\title{
Electron heating in metallic resistors at sub-Kelvin temperature
}

\author{
B. Huard, H. Pothier, and D. Esteve \\ Quantronics group, Service de Physique de l'Etat Condensé (CNRS URA 2464), \\ DRECAM, CEA-Saclay, 91191 Gif-sur-Yvette, France \\ K. E. Nagaev \\ Institute of Radioengineering and Electronics, Russian Academy of Sciences, Mokhovaya ulica 11, 125009 Moscow, Russia
}

(Dated: August 24, 2021)

\begin{abstract}
In the presence of Joule heating, the electronic temperature in a metallic resistor placed at subKelvin temperatures can significantly exceed the phonon temperature. Electron cooling proceeds mainly through two processes: electronic diffusion to and from the connecting wires and electronphonon coupling. The goal of this paper is to present a general solution of the problem, in a form that can easily be used in practical situations. As an application, we compute two quantities that depend on the electronic temperature profile: the second and the third cumulant of the current noise at zero frequency, as a function of the voltage across the resistor. We also consider time dependent heating, an issue relevant for experiments in which current pulses are used, for instance in time-resolved calorimetry experiments.
\end{abstract}

PACS numbers: 72.15.Lh,73.50.-h,73.23.-b,73.50.+d,72.70.+m

\section{MOTIVATIONS AND OUTLINE}

When performing electrical measurements, the signal to noise ratio can usually be improved by simply increasing the currents or voltages. In low-temperature experiments, this procedure is problematic because of Joule heating, which can affect the temperature of the circuit under investigation or the temperature of resistors on bias lines, leading to excess noise. Particularly critical is the situation in the sub-Kelvin range, because the temperature of the electrons decouples from the lattice temperature 1,2,3.4. A very conservative, wide-spread rule of thumb among experimentalists is that the voltage $V$ across the small conductors should not exceed $k_{B} T_{\mathrm{ph}} / e$, with $T_{\mathrm{ph}}$ the lattice (phonon) temperature. In contrast, macroscopic components, like commercial resistors, are believed to be immune to electron heating. In fact, the first rule is severe, and the second assertion is often incorrect. The goal of the present article is to provide the experimental physicist with easy evaluation tools of heating effects, in order to optimize experiments.

The important parameters are the voltage $V$, the resistance $R$, the lattice temperature $T_{\mathrm{ph}}$, the resistor volume $\Omega$ and a parameter $\Sigma$ that describes electron-phonon coupling. The first step is to calculate the characteristic temperature $T_{\Sigma}$, which is the temperature that the electrons would reach if cooling would occur only through the coupling to a bath of zero-temperature phonons:

$$
T_{\Sigma}=\left(\frac{V^{2}}{\Sigma \Omega R}\right)^{1 / 5}
$$

The average electron temperature can then be directly read from Fig.2, the central result of this work, in which the voltage $V$, the average temperature $T_{\mathrm{av}}$ and the lattice temperature $T_{\mathrm{ph}}$ are all expressed in units of $T_{\Sigma}$. In the section IIA, we explain how this result is obtained, and give analytical expressions in various limits. The results are used to calculate the second and third cumulant of the current noise produced by the resistor (section IIB, Fig.(4). Numerical applications are carried out explicitely in section IIC, showing in particular that heating in commercial resistors can be important. In section III, we address time-dependent situations, and calculate how fast electrons heat up in a resistor when a current is applied, and how fast they cool down when the current is switched off. For small voltages $\left(e V \ll k_{B} T_{\Sigma}\right)$, the variations of temperature in both transients is exponential, with the diffusion time $\tau_{D}$ across the whole conductor as a characteristic time (see Fig. 55). In the opposite limit $\left(e V \gg k_{B} T_{\Sigma}\right)$, heating is exponential, but cooling proceeds very slowly, with a powerlaw dependence (see Fig.60). The timescale is the electron-phonon scattering time $\tau_{\mathrm{e}-\mathrm{ph}}$ at temperature $T_{\Sigma}$, defined by Eq. (17). As a numerical application, we consider in section IIIC a situation where repeated current pulses are applied to a resistor, and compute the time-dependence of the electron temperature (Fig.77).

\section{STATIONARY SITUATIONS}

\section{A. Solution of the heat equation}

When a voltage $V$ is applied to a two-terminal resistor (see top of Fig.(1), the Joule power $V^{2} / R$ is delivered to the electrons. This power can dissipate by two mechanisms: the first one, which dominates at room temperature or in macroscopic resistors, is phonon emission. It follows, at temperatures well below the Debye temperature, a $T^{n}(x)-T_{\mathrm{ph}}^{n}$ dependence, with $4 \leq n \leq 6$, and $T(x)$ the local electron temperature, $T_{\mathrm{ph}}$ the phonon temperature ${ }^{\underline{5}}$. The second mechanism is the simple diffu- 
sion of the energetic electrons out of the resistor. The energy is then dissipated in the connecting leads, which are, in typical situations, large and low-resistive. The balance between the Joule power and the two cooling mechanisms can be expressed in the form of a heat equation 6

$$
\frac{d}{d x}\left(\frac{L_{o} T(x)}{R} \frac{d}{d x} T(x)\right)=-\frac{V^{2}}{R}+\Sigma \Omega\left(T^{5}(x)-T_{\mathrm{ph}}^{5}\right)
$$

with $x$ the position along the resistor in reduced units ( $x$ runs from 0 to 1 ), $L_{o}=\pi^{2} k_{B}^{2} / 3 e^{2}$ the Lorenz number, $\Omega$ the resistor volume, $\Sigma$ the electron-phonon coupling constant (typically $\Sigma \simeq 2 \mathrm{nW} / \mu \mathrm{m}^{3} / \mathrm{K}^{5}$ for good metals $\left.{ }^{7}\right)$. The left hand side of Eq. (2) accounts for heat transport by electron diffusion, which is expressed by the Wiedemann-Franz law, stating that the electron thermal conductivity is proportional to the product of the electrical conductivity and the electron temperature. The following assumptions have been made to write Eq. (2):

1. The electron temperature $T(x)$ is assumed to be well defined locally, i.e. the local electron energy distribution function is a Fermi function. This requires that the thermalization of electrons among themselves (e.g. by Coulomb interaction) occurs faster than the diffusion of electrons across the resistor ${ }^{8}$, a condition usually obeyed except for short wires (length $\lesssim 50 \mu \mathrm{m}$ ) made of very pure materials ${ }^{9}$.

2. The last term of the equation, which describes cooling by phonons, assumes that the lattice temperature $T_{\mathrm{ph}}$ does not depend on the local electron temperature $T(x)$. Corrections due to the Kapitza resistance between the phonons of the resistive film and the substrate could in principle be included ${ }^{3.10}$, but their contribution is not essential in practice.

3. The heat power transfered to phonons was taken proportional to $T^{n}(x)-T_{\mathrm{ph}}^{n}$ with $n=5$. Theoretically, it is predicted that the exponent $n$, which is related to a $E^{2-n}$ dependence of the electronphonon scattering rate with electron energy $E$, can range from 4 to 6 , depending on the relative sizes of the thermal phonon wavelength and the electron mean free path, on the dimensionality of the phonon system, and on the dynamics of impurities ${ }^{5}$. In most experiments, values close to $n=5$ have been found (see discussions in Refs. 5,7.11), therefore our choice. The calculations can however be easily extended to other values of $n$, and the results presented in Fig.2 apply, with another definition of $T_{\Sigma}$, as discussed in the following.

4. Radiative cooling $\frac{11}{11}$, which has a negligible effect in resistors connected to large, non-superconducting $\operatorname{contacts}^{12}$, is neglected.
The heat equation (2) has to be solved with boundary conditions for $T(x)$ at $x=0$ and $x=1$. When the connecting wires to the resistor are low-resistive and very large compared to the resistor, as is the case for macroscopic resistors made of thin and narrow metallic stripes of metal, one can assume $T(0)=T(1)=T_{\mathrm{ph}}$. This simple hypothesis will be made in the following. For on-chip thin-film resistors, heating of the contact pads themselves may however not be negligible $\frac{13}{3}$.

Before a general solution of Eq. (2) is presented, we recall simple limits. The so-called interacting hot-electron limit $\underline{\underline{6}}$ is obtained by neglecting phonon cooling:

$$
T(x)=\sqrt{T_{\mathrm{ph}}^{2}+\frac{3}{\pi^{2}} x(1-x)\left(\frac{e V}{k_{B}}\right)^{2}}
$$

(see left panel of Fig.1, dashed lines). For $T_{\mathrm{ph}}=0$, the maximal temperature is $T\left(\frac{1}{2}\right)=(\sqrt{3} / 2 \pi) \mathrm{eV} / k_{B} \simeq$ $0.28 \mathrm{eV} / k_{B}$, and the average temperature is $T_{\mathrm{av}}=$ $\int_{0}^{1} T(x) d x=(\sqrt{3} / 8) e V / k_{B} \simeq 0.22 \mathrm{eV} / k_{B}$. Electronphonon coupling further reduces the temperature, so that this is an upper bound on the average electron temperature, which numerically reads, keeping $\frac{14}{\underline{14}}$ now $T_{\mathrm{ph}}$ :

$$
\frac{T_{\mathrm{av}}}{T_{\mathrm{ph}}} \leq \sqrt{1+\left(0.22 \frac{e V}{k_{B} T_{\mathrm{ph}}}\right)^{2}} .
$$

In particular, the rule of thumb $\mathrm{eV}=k_{B} T_{\mathrm{ph}}$ corresponds to a $2.5 \%$ average overheating of the electrons. Numerically, one obtains the equivalent expression

$$
T_{\mathrm{av}}[\mathrm{mK}] \leq \sqrt{T_{\mathrm{ph}}^{2}[\mathrm{mK}]+(2.5 \times V[\mu \mathrm{V}])^{2}} .
$$

In the opposite limit where cooling by diffusion can be neglected, the electron temperature is homogeneous and equal to ${ }^{3}$

$$
T=\left(T_{\mathrm{ph}}^{5}+T_{\Sigma}^{5}\right)^{1 / 5} .
$$

In the following, we call this limit "the fully thermalized regime". In the limit $T_{\mathrm{ph}}=0$, the temperature grows as $V^{2 / 5}$ (see Fig. 3).

In intermediate regimes, the temperature profile is obtained by solving numerically the heat equation. For generality, it is convenient to rewrite it in reduced units. One possibility is to take as a reference the "cross-over temperature" $\underline{\underline{4}} T_{\mathrm{co}}=\left(\Sigma \Omega R e^{2} / k_{B}^{2}\right)^{-1 / 3}$, which is the energy scale for which phonon cooling and diffusion cooling are equaly important. This temperature is an intrinsic quantity for the resistor, which in particular does not depend on $V$ or $T_{\mathrm{ph}}$. However, it does not correspond to the electron temperature in any limit, therefore we prefer to take as a reference $T_{\Sigma}$, keeping in mind that it depends on $V$. Defining $\theta(x)=T(x) / T_{\Sigma}, v=e V / k_{B} T_{\Sigma}$ $\left(=\left(e V / k_{B} T_{\mathrm{co}}\right)^{3 / 5}\right), \theta_{\mathrm{ph}}=T_{\mathrm{ph}} / T_{\Sigma}\left(\propto V^{-2 / 5}\right)$, Eq. (22) reads:

$$
\frac{d^{2}}{d x^{2}} \theta^{2}(x)=\frac{6}{\pi^{2}} v^{2}\left(\theta^{5}(x)-\theta_{\mathrm{ph}}^{5}-1\right) .
$$


The temperature profile being symmetric with respect to the middle of the wire, Eq. (7) needs to be solved for $0<x<\frac{1}{2}$ with the boundary conditions $\theta(0)=\theta_{\mathrm{ph}}$ and $\theta^{\prime}\left(\frac{1}{2}\right)=0$. Instead of solving this non-linear differential equation with boundary conditions specified at different points, it is convenient to rewrite it in the following integral form:

$\frac{2 \sqrt{3} v}{\pi \theta_{m}} x=\int_{\theta_{\mathrm{ph}}^{2} / \theta_{m}^{2}}^{\theta^{2}(x) / \theta_{m}^{2}} \mathrm{~d} u\left(\frac{2}{7} \theta_{m}^{5}\left(u^{7 / 2}-1\right)-\lambda^{5}(u-1)\right)^{-1 / 2}$

with $\theta_{m}=\theta\left(\frac{1}{2}\right)$ and $\lambda=\left(\theta_{\mathrm{ph}}^{5}+1\right)^{1 / 5}$. The value of $\theta_{m}$ is obtained by solving Eq. (8) for $x=1 / 2$. In the left panel of Fig.1, the temperature profile along the resistor is given for $v=1,2,3,10$ and 30 , assuming $T_{\mathrm{ph}}=0$. At $v \lesssim 1$, one recovers the result of Eq. (3), plotted as dashed lines: phonon cooling can be neglected. For $v \gtrsim 10$, it is an excellent approximation to take $\theta_{m}=\lambda$ in Eq. (8), a value that does not depend on $v . T(x)$ is then a function of $x v$ only, which is essentially constant around the middle of the wire, whereas at a distance $5 / v$ from the contacts one obtains the profiles shown in the right panel of Fig.1 for $\theta_{\mathrm{ph}}=0$ (see note 15), 0.5 and 1 . The characteristic length over which the electron temperature varies from $T_{\mathrm{ph}}$ to $T_{\Sigma}$ is therefore $L_{\Sigma}=L / v=\frac{\sqrt{3}}{8} L^{3 / 5} L_{\mathrm{e}-\mathrm{ph}}^{2 / 5}$, where the "electron-phonon length"

$$
L_{\mathrm{e}-\mathrm{ph}}=\left(\frac{8 k_{B}}{\sqrt{3} e}\right)^{5 / 2}\left(\rho \Sigma V^{3}\right)^{-1 / 2}
$$

( $\rho$ being the resistivity) is defined, following 16 Ref. 6 , as the resistor length for which $4 k_{B} T_{\Sigma} / R$ is equal to the current noise in the interacting hot-electron regime $\frac{\sqrt{3}}{2} e I$. This length is typically of the order of a few $\mu \mathrm{m}$ for voltages of the order ot $1 \mathrm{mV}$.

From the complete temperature profile $\theta(x)$, the average electron temperature $T_{\mathrm{av}}$ is obtained using $T_{\mathrm{av}}=$ $T_{\Sigma} \int_{0}^{1} \theta(x) \mathrm{d} x$. The central result of this work is the resulting plot, shown in Fig. 2, of the average temperature $T_{\text {av }}$ as a function of the voltage $V$, both in units of $T_{\Sigma}$, for $T_{\mathrm{ph}} / T_{\Sigma}=0,0.25,0.5,0.75$ and 1 . At $\theta_{\mathrm{ph}}=0$ and $v \lesssim 2, T_{\mathrm{av}} \sim \frac{\sqrt{3}}{8} \frac{\mathrm{eV}}{k_{B}}$ (dotted line), whereas for $v \gtrsim 4$, $T_{\mathrm{av}} / T_{\Sigma} \approx 1-1.16 / v$ (dashed line). This $1 / v$ dependence is due to the crossover regions of width $\sim 5 / v$ at the resistor ends. Figure 2 can be directly used to read out the average electron temperature $T_{\mathrm{av}}$ for a given set of experimental parameters $\left(V, T_{\mathrm{ph}}\right)$, after having computed $T_{\Sigma}$ with Eq. (11). Interestingly, the corresponding curves for other exponents of the temperature in the last term of Eq. (2) ( $n=4$ or 6 instead of 5) are almost identical (see inset), and the same curves can be used to evaluate $T_{\mathrm{av}}$, however with the generalized definition of the reference temperature $T_{\Sigma}=\left(V^{2} / \Sigma \Omega R\right)^{1 / n}$.

However, because of the use of reduced units which depend on $V$, the $v$-dependence of $T_{\text {av }}$ at a fixed value of $\theta_{\mathrm{ph}}$ shown in Fig.2 does not correspond to a situation in which $V$ is changed at a fixed $T_{\mathrm{ph}}$, since $\theta_{\mathrm{ph}} \propto V^{-2 / 5}$. In order to visualize how temperature increases with $V$ at a given phonon temperature, we plot in Fig. 3 the average temperature $T_{\mathrm{av}}(V)$ for various $T_{\mathrm{ph}}$, with $V$ and $T_{\mathrm{ph}}$ given in units of $T_{\mathrm{co}}=\left(\Sigma \Omega R e^{2} / k_{B}^{2}\right)^{-1 / 3}$, which is constant for a given resistor. The range in voltage $V$ is the same as in Fig.22 We have used the relations $v^{5 / 3}=e V / k T_{\mathrm{co}} ;\left(T_{\mathrm{av}} / T_{\Sigma}\right) v^{2 / 3}=T_{\mathrm{av}} / T_{\mathrm{co}}$.

\section{B. Second and third cumulants of the current noise}

\section{at zero frequency}

The temperature profile can be used to evaluate the current noise properties of the resistor. We focus here on the second $\left(S_{2}\right)$ and third $\left(S_{3}\right)$ cumulants of noise at low frequencies $\left(\hbar \omega \ll e V\right.$ for $S_{2}, \hbar \omega \ll e V, \hbar / \tau_{D}$ for $S_{3}$ (see Ref.17), with $\tau_{D}=L^{2} / D$ the diffusion time, $D$ the diffusion constant):

$$
S_{2}=2 \iint \mathrm{d} t\langle\delta I(0) \delta I(t)\rangle
$$

and

$$
S_{3}=\iint \mathrm{d} t_{1} \mathrm{~d} t_{2}\left\langle\delta I(0) \delta I\left(t_{1}\right) \delta I\left(t_{2}\right)\right\rangle
$$

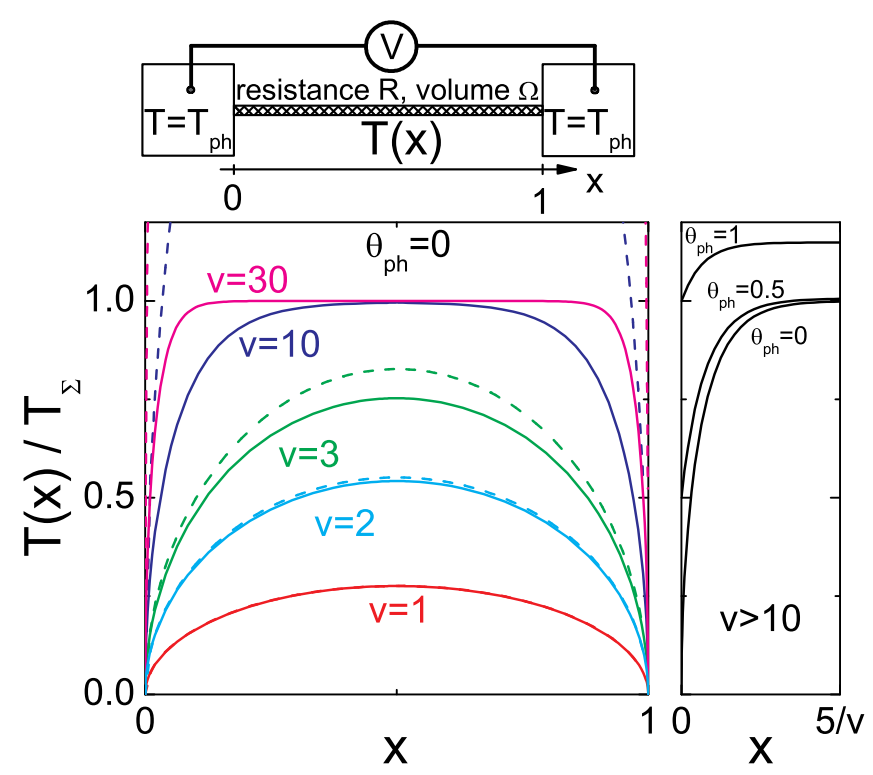

FIG. 1: (Color online) Top: Resistor biased by a voltage $V$ and placed between two connecting wires in which the electron temperature $T$ and the phonon temperature $T_{\mathrm{ph}}$ are equal. Left panel: Solid lines: temperature profile in the resistor for different values of $v=e V / k_{B} T_{\Sigma}$ with $T_{\Sigma}=$ $\left(V^{2} / \Sigma \Omega R\right)^{1 / 5}$. Dashed lines: temperature profile expected when phonon cooling is neglected (Eq. (3) ). Right panel: temperature profile near the ends of the resistor for $v>10$ and $\theta_{\mathrm{ph}}=T_{\mathrm{ph}} / T_{\Sigma}=0,0.5,1$. 
with $\delta I(t)=I(t)-\langle I\rangle$. It has been shown that when phonon cooling can be disregarded and $\mathrm{eV} \gg k_{B} T_{\mathrm{ph}}, S_{2}$ and $S_{3}$ are proportional to the applied current $\frac{6,13}{}$ : $S_{2}=$ $F_{2} \times 2 e I$ and $S_{3}=F_{3} \times e^{2} I$ with $F_{2}$ and $F_{3}$ generalized "Fano factors". When furthermore the rate of electronelectron interaction is negligible compared to $1 / \tau_{D}$, the distribution function is not a Fermi function, but a function with two steps 8,18 , and $\frac{18,19,20}{12} F_{2}=\frac{1}{3} \approx 0.33$; $F_{3}=\frac{1}{15} \approx 0.067$. In the opposite limit, where electronelectron interaction is strong, electrons thermalize locally to distribute in energy according a Fermi function, and the temperature profile is given by Eq. (3). One then obtains $^{22,35} F_{2}=\frac{\sqrt{3}}{4} \approx 0.43$ and $^{21} F_{3}=\frac{8}{\pi^{2}}-\frac{9}{16} \approx 0.248$.

In presence of strong phonon cooling $(v \gg 1)$, the electron temperature becomes homogeneous, at a value $T_{\Sigma}$ smaller than $\mathrm{eV} / k_{B}$. It is then expected ${ }^{21}$ that $F_{2}, F_{3} \rightarrow$ 0 .

For intermediate coupling to phonons, $F_{2}$ and $F_{3}$ depend on the voltage across the resistor. Their value are obtained from the full solution of the heat equation: the second cumulant is given by a Johnson-Nyquist-like formula ${ }^{6} S_{2}=4 k_{B} T_{\mathrm{av}} / R$ in which the noise temperature is the average electron temperature $T_{\mathrm{av}}$, yielding $F_{2}=2 k_{B} T_{\mathrm{av}} / \mathrm{eV}=2 \theta_{\mathrm{av}} / v$. This formula can be understood as resulting from the added Johnson-Nyquist noise of small sections of the resistor, each at a temperature $T(x)$. The decay of $F_{2}$ at large $V$ was discussed in Ref. 22 , and the complete crossover was calculated in Ref. 23 by numerical integration of Eq. (2). In turn, $F_{3}$

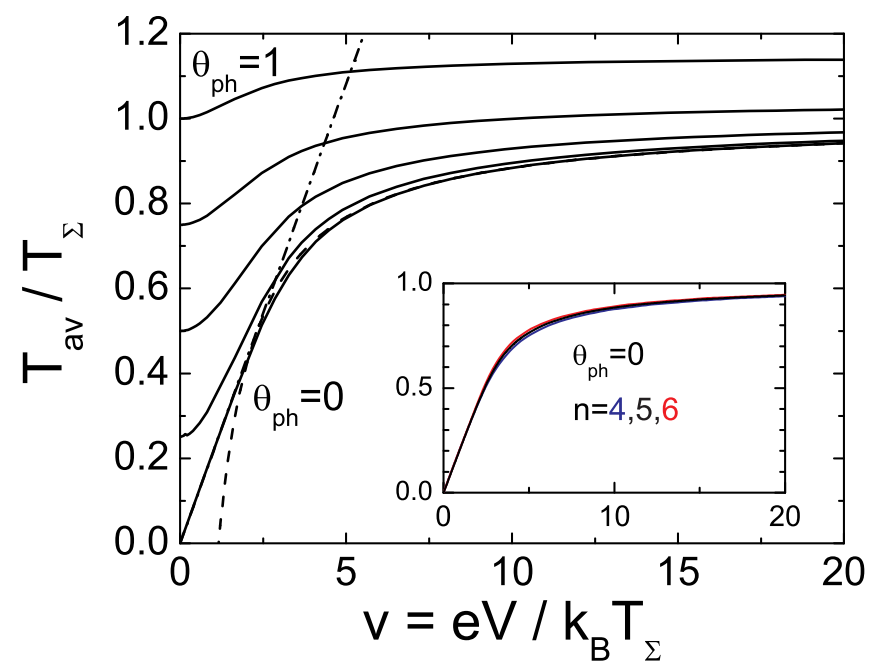

FIG. 2: (Color online) Average temperature $T_{a v}$ in units of $T_{\Sigma}$, as a function of $v=e V / k_{B} T_{\Sigma}$, for $\theta_{p h}=T_{p h} / T_{\Sigma}=0$, $0.25,0.5,0.75,1$ (from bottom to top). The dotted line corresponds to the low- $v$ approximation $T_{a v}=\frac{\sqrt{3}}{8} \frac{e V}{k_{B}}$, the dasheddotted line to the large- $v$ approximation $T_{a v}=1-1.16 / v$. Inset: at $\theta_{p h}=T_{p h} / T_{\Sigma}=0$, comparison of the evolution of the average temperature with $v$ for various exponents $n$ of the temperature in the expression of the heat flow through electron-phonon coupling. is given by (see Appendix)

$$
F_{3}=\frac{36}{\pi^{2}} \int_{0}^{1} \mathrm{~d} x \mathrm{~d} y \frac{1}{\theta(x)} G_{1}(\theta, x, y)\left\{\theta(y)-2 \theta_{\mathrm{av}}\right\}
$$

where $G_{1}(\theta, x, y)$ the Green's function such that $\left(\nabla^{2}+\frac{15}{\pi^{2}} v^{2} \theta^{3}(x)\right) G_{1}(\theta, x, y)=\delta(x-y)$ and $G_{1}(\theta, 0, y)=G_{1}(\theta, x, 0)=0$. The calculation of $F_{3}$ is detailed in the Appendix. The right panel of Fig.4 shows the voltage dependence of $F_{2}$ (blue line) and $F_{3}$ (red line) as a function of $v$ (bottom axis) and $L / L_{\mathrm{e}-\mathrm{ph}}=(\sqrt{3} v / 8)^{5 / 2}$ (top axis), for $T_{\mathrm{ph}}=0$. Also shown with a dashed line is the curve obtained for $F_{2}$ when electron diffusion is neglected ${ }^{22}$, using Eq. (6) (dashed line), which gives $F_{2}=2 / v \propto V^{-3 / 5}$. In turn, at large voltages, $F_{3} \propto v^{-2} \propto V^{-6 / 5}$. If one considers a situation where the resistor length $L$ is varied at constant current, then $F_{2} \propto 1 / L$ and $F_{3} \propto 1 / L^{2}$. The decay to zero of $F_{2}$ and $F_{3}$ from the interacting hot-electron values $\left(\frac{\sqrt{3}}{4}\right.$ and $\left.\frac{8}{\pi^{2}}-\frac{9}{16}\right)$ is therefore very slow, as already pointed in Ref. 21, 23. The non-Gaussian character of the current noise, evidenced by $F_{3} \neq 0$, is washed out at $L / L_{\mathrm{e}-\mathrm{ph}} \gtrsim 10$.

\section{Examples}

We consider here a few cases illustrating the use of the results given in the preceding sections. As a first example, we consider a $10 \mu \mathrm{m}$-long, $100 \mathrm{~nm}$-wide and $5 \mathrm{~nm}$-thick $\mathrm{Cr}$ resistor with resistance $R=25 \mathrm{k} \Omega$ like these used in Ref.25, biased at $V=1 \mathrm{mV}$ and placed at

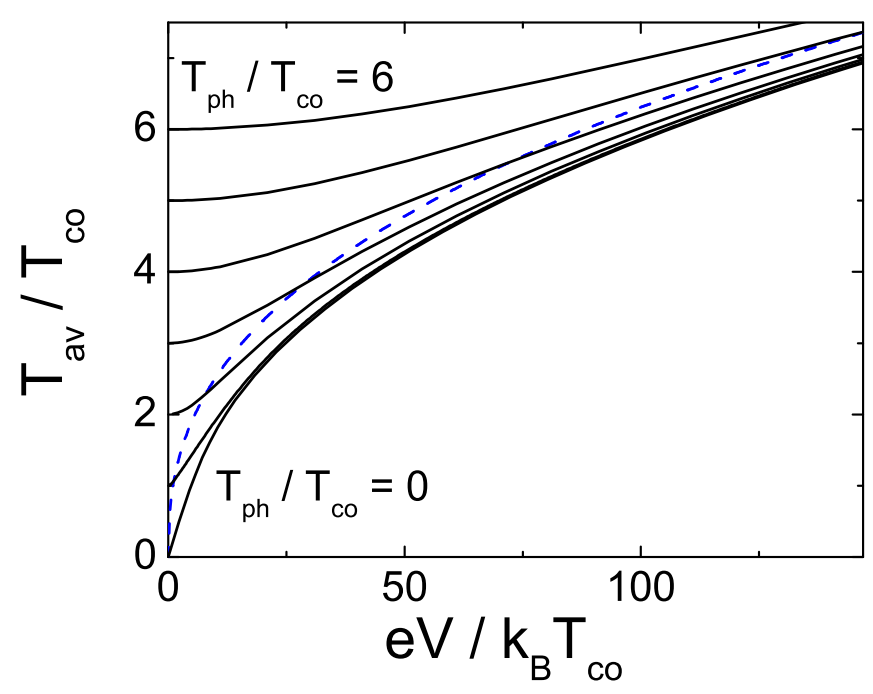

FIG. 3: (Color online) Average temperature $T_{a v}$ as a function of voltage $V$, for various temperatures $T_{\mathrm{ph}}$, all in units of $T_{c o}=\left(\Sigma \Omega R e^{2} / k_{B}^{2}\right)^{-1 / 3}$. The value of $T_{\mathrm{ph}} / T_{c o}$ is given by the intersection of the curves with the vertical axis. Blue dashed line is the reference temperature $T_{\Sigma}$. 
$T_{\mathrm{ph}}=20 \mathrm{mK}$. Assuming $\Sigma_{\mathrm{Cr}}=2 \mathrm{nW} / \mu \mathrm{m}^{3} / \mathrm{K}^{5}$, the characteristic temperature is $T_{\Sigma}=1.3 \mathrm{~K}$, and the voltage and phonon temperature in reduced units $v=e V / k_{B} T_{\Sigma} \sim 9$ and $\theta_{\mathrm{ph}}=0.015$. The noise temperature is directly read from Fig.2 $T_{\mathrm{av}} \sim 0.87 \times T_{\Sigma} \sim 1.15 \mathrm{~K}$. At this voltage, heating of the resistor is thus very important, an effect which hindered the authors of Ref. 25 from drawing clearcut conclusions from Coulomb blockade measurements at finite voltage. Increasing the resistor volume $\Omega$ with "cooling fins" can help decreasing electron heating $\underline{3}$, but such a procedure is extremely inefficient since the characteristic temperature $T_{\Sigma}$ decreases as $\Omega^{-1 / 5}$ only.

As a second application, we now consider a commercial macroscopic surface mount resistor, with $R=$ $500 \Omega$. Such resistors, made of thin $(\sim 10 \mathrm{~nm}) \mathrm{NiCr}$ films ${ }^{26}$ with resistivity $\rho \sim 100 \mu \Omega \mathrm{cm}$ and dimensions $\sim 1 \mathrm{~mm} \times 0.2 \mathrm{~mm}$, were used as bias resistors in measurements of the state of superconducting Josephson Q-bits27.28 performed at $15 \mathrm{mK}$, with a bias current $\sim 0.8 \mu \mathrm{A}$, resulting in a voltage $V \sim 400 \mu \mathrm{V}$. The corresponding temperature scale $T_{\Sigma} \sim 150 \mathrm{mK}$ yields $v=e V / k_{B} T_{\Sigma} \sim 30$ and $\theta_{\mathrm{ph}} \sim 0.1$, hence, from Fig.2, $T_{\mathrm{av}} \sim T_{\Sigma} \sim 150 \mathrm{mK}$. Even in such a macroscopic resistor, the volume is not sufficient to provide with enough electron-phonon coupling, and heating is important. In the next section, we show how this heating is limited when pulses are used instead of static voltages.

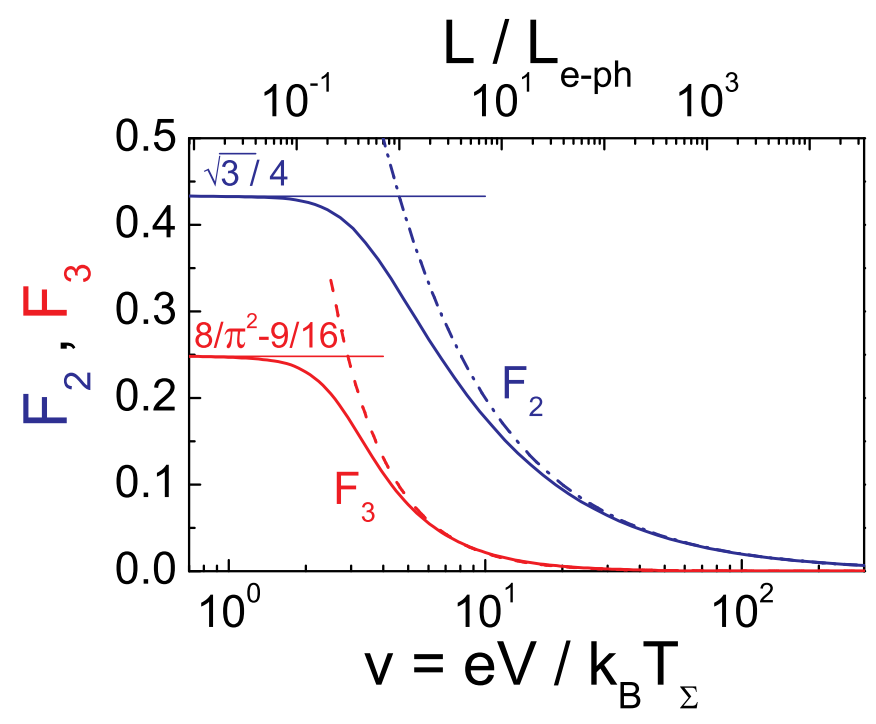

FIG. 4: (Color online) Solid lines: Fano factors $F_{2}=S_{2} / 2 e I$ (blue) and $F_{3}=S_{3} / e^{2} I$ (red) for the zero-frequency second and third cumulant of noise, from the hot electron limit to the fully thermalized regime. Dashed-dotted line: asymptotic dependence of $F_{2}$ neglecting electron diffusion: $F_{2}=2 / v$. Top axis is resistor length over $L_{\mathrm{e}-\mathrm{ph}}$ (see Eq. (9)).

\section{TIME-DEPENDENT SITUATIONS: SWITCHING ON AND OFF JOULE HEATING}

The case of a constant voltage $V$ across the resistor, which was investigated above, can be extended to the case of slowly varying voltages directly. However, when $V$ changes on timescales shorter than the diffusion time or than the electron-phonon scattering time ${ }^{29}$ (see below), the previous results cannot be used to calculate instantaneous temperatures. These issues are solved by adding to the heat equation (2) a time-dependent term $d Q / d t=C_{e} d T / d t$, with $C_{e}=\gamma T \Omega$ the electronic heat capacity, $\gamma=\left(\pi^{2} / 3\right) k_{B}^{2} \nu_{F}$ (from Fermi liquid theory), with $\nu_{F}$ the density of states at Fermi energy (spin degeneracy included). When $V(t)=V f(t)$, the time-dependent heat equation can then be rewritten, in reduced units, as

$$
\frac{\partial \theta^{2}}{\partial \tau}=\frac{\partial^{2} \theta^{2}}{\partial x^{2}}-\frac{6}{\pi^{2}} v^{2}\left(\theta^{5}-\theta_{\mathrm{ph}}^{5}-f^{2}(\tau)\right)
$$

where $\tau=t / \tau_{D}$ is the reduced time with $\tau_{D}=L^{2} / D$ the diffusion time. Note that the reference temperature $T_{\Sigma}$ used to define $\theta(x)=T(x) / T_{\Sigma}$ is calculated with the voltage scale $V$, not with the time-dependent value $V(t)$. In the following, we treat more explicitely two situations: how a resistor heats up when the voltage is applied at $t=0$, i.e. $f(\tau)=H(\tau)$, and how a resitor cools down when the voltage is set to zero at $t=0$, i.e. $f(\tau)=1-H(\tau)$. Here, $H(\tau)$ is the Heaviside function ( 0 for $\tau<0,1$ for $\tau>0$ ). These situations also allow to describe experiments in which current or voltage pulses are used like, for example, when measuring the switching rate of Josephson junctions 27 . Understanding how the pulse characteristics can reduce the noise in such measurements is therefore important to design the readout of superconducting Q-bits.

When a voltage is applied, the linear drop of the electrical potential, which results from the collective charge modes, establishes after an $R C$ time, where the capacitance $C$ is the capacitance of the wire to ground. This time is generally much shorter than the time necessary to build up the temperature profile, which involves diffusion of individual electrons. Hence, we consider here that Joule heating is homogeneous as soon as a voltage is applied. When $v \lesssim 1$, the temperature profile is entirely determined by the temperature at the ends of the resistor, therefore a steady-state regime is reached only when the electrons have diffused across the whole resistor and the characteristic time is the diffusion time $\tau_{D}$. If $v \gg 1$, the transient is shorter because, apart from very close to the ends, the temperature is mostly determined by a local equilibrium between Joule heating and phonon emission. We now treat quantitatively these two limits. 


\section{A. Small $v$ limit}

If $v \lesssim 1, \theta(x, t)-\theta_{\mathrm{ph}} \ll 1$ even when the stationary regime is reached, and Eq. (11) reduces to

$$
\frac{\partial \theta^{2}}{\partial \tau}=\frac{\partial^{2} \theta^{2}}{\partial x^{2}}+\frac{6}{\pi^{2}} v^{2} f^{2}(\tau)
$$

As shown in section IIA, the proper energy scale when $v \lesssim 1$ is $e V$, and the solution of Eq. (12) that satisfies the boundary conditions $T(0, \tau)=T(1, \tau)=T_{\mathrm{ph}}$ reads

$$
\left(\frac{k_{B} T(x, \tau)}{e V}\right)^{2}=\left(\frac{k_{B} T_{\mathrm{ph}}}{e V}\right)^{2}+\sum_{k \text { odd }} a_{k}(\tau) \sin (\pi k x)
$$

with $a_{k}(\tau)$ solution of

$$
\frac{\mathrm{d} a_{k}(\tau)}{\mathrm{d} \tau}+\pi^{2} k^{2} a_{k}(\tau)=\frac{24}{\pi^{3} k} f^{2}(\tau) .
$$

In the case where $f(\tau)=H(\tau)$, heating is then given by $a_{k}(\tau)=\left(24 / \pi^{5} k^{3}\right)\left(1-e^{-\pi^{2} k^{2} \tau}\right)$, and in the case $f(\tau)=1-H(\tau)$, cooling from the profile (3) follows $a_{k}(\tau)=\left(24 / \pi^{5} k^{3}\right) e^{-\pi^{2} k^{2} \tau}$. Corresponding temperature profiles at various times are plotted in the top panels of Fig. 5 assuming $T_{\mathrm{ph}}=0$, whereas the time evolution of the average temperature $T_{\mathrm{av}}$ is plotted in the bottom panels. At very short times, the average temperature grows as $k_{B} T_{\mathrm{av}} / e V=\sqrt{6 \tau} / \pi$. At $\tau \gtrsim 0.01$, a better approximation is

$$
\frac{k_{B} T_{\mathrm{av}}}{e V} \simeq \frac{\sqrt{3}}{8} \sqrt{1-\exp (-10 \tau)}
$$

which cannot be distinguished from the exact solution in Fig. 5. At $\tau \simeq 0.5$, the asymptotical temperature profile given by Eq. (2) is essentially established. Conversely, after the voltage is turned off, the temperature decay is well approximated (within the line width in Fig.5) by

$$
\frac{k_{B} T_{\mathrm{av}}}{e V} \simeq \frac{\sqrt{3}}{8} \exp (-5 \tau)
$$

Hence, in the low-voltage regime, heating and cooling occur exponentially, and the timescale is the diffusion time $\tau_{D}$. In the example of the $500 \Omega$ commercial resistor in section IIB, $\tau_{D} \sim 3 \mathrm{~ms}$. For metallic wires made of pure materials 26 with an elastic mean free path of the order of $40 \mathrm{~cm}^{2} / \mathrm{s}, \tau_{D} \sim 20 \mathrm{~ns}$ for a length $L \sim 20 \mu \mathrm{m}$.

\section{B. Large $v$ limit}

If $v \gg 1$, it was shown in section IIA that the temperature becomes almost homogeneous in the wire. The relevant timescale is then the electron-phonon scattering time $\frac{11,30,33}{}$ at the characteristic temperature $T_{\Sigma}$ :

$$
\tau_{\mathrm{e}-\mathrm{ph}}\left(T_{\Sigma}\right)=\frac{\gamma}{\Sigma T_{\Sigma}^{3}}=\frac{\pi^{2}}{3} \frac{L_{\Sigma}^{2}}{D}
$$

with $L_{\Sigma}$ the characteristic length for the variation of $T(x)$ introduced in section IIA. Numerically, $\gamma / \Sigma \approx$ $0.03 \mu \mathrm{s} \mathrm{K} \mathrm{K}^{3}$. Using $\frac{6}{\pi^{2}} v^{2} \tau=2 \tau^{*}$ with

$$
\tau^{*}=t / \tau_{\mathrm{e}-\mathrm{ph}}\left(T_{\Sigma}\right)
$$

Eq. (11) reduces to

$$
\frac{\partial \theta^{2}}{\partial \tau^{*}}=-2\left(\theta^{5}-\theta_{\mathrm{ph}}^{5}-f^{2}\left(\tau^{*}\right)\right)
$$

which for $\theta_{\mathrm{ph}}=f=0$ is simply equivalent to

$$
\frac{\partial T}{\partial t}=-\frac{T}{\tau_{\mathrm{e}-\mathrm{ph}}(T)}
$$

expressing that the instantaneous decay rate of $T$ is exponential with a characteristic time $\tau_{\mathrm{e}-\mathrm{ph}}(T)$. Equation (19) yields

$$
\int_{\theta^{2}(0)}^{\theta^{2}\left(\tau^{*}\right)} \frac{\mathrm{d} w}{f^{2}\left(\tau^{*}\right)+\theta_{\mathrm{ph}}^{5}-w^{5 / 2}}=2 \tau^{*} .
$$

When $f(\tau)=1-H(\tau)$ and $T_{\mathrm{ph}}=0$, the temperature decay from $T_{\Sigma}$ has a simple form:

$$
\theta\left(\tau^{*}\right)=\left(1+3 \tau^{*}\right)^{-1 / 3}
$$

This temperature decay, which was directly measured in Ref.11, follows a power law only, so that it takes

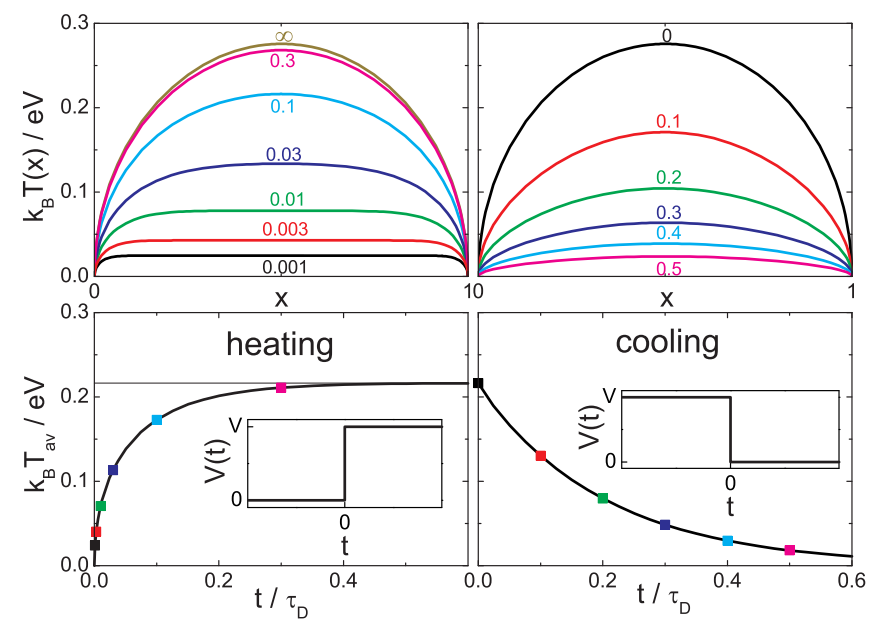

FIG. 5: (Color online) Time evolution of the temperature profile (top panels) and of the average temperature $T_{a v}$ (bottom panels) in the limit $v \ll 1$, for $T_{p h}=0$. Left panels: heating sequence of the resistor when $V(t) / V=H(t)$ (as shown in inset); right panels: cooling sequence of the resistor when $V(t) / V=1-H(t)$ (as shown in inset). The profiles are plotted at various values of $t / \tau_{D}$, with $\tau_{D}=L^{2} / D$ the diffusion time. The colors of the solid curves in the top panels corresponds to those of the square symbols in the bottom panels. The curves in the bottom panels cannot be distinguished from $k_{B} T_{a v} / e V \simeq \frac{\sqrt{3}}{8} \sqrt{1-\exp (-10 \tau)}$ and $\frac{\sqrt{3}}{8} \exp (-5 \tau)$, respectively. 
a very long time to recover the base temperature after the voltage is set to 0 , which is due to the divergence of $\tau_{\mathrm{e}-\mathrm{ph}}(T)$ when $T \rightarrow 0$. The results of Eq. (21) with $f=1$ (heating) and $f=0$ (cooling) are plotted in Fig.6 in the case $T_{\mathrm{ph}}=0$, with linear (top) and logarithmic (bottom) time scales. The temperature rise is well approximated by $\theta\left(\tau^{*}\right) \approx \sqrt{2 \tau^{*}}$ when $\tau^{*} \lesssim 0.2$ (dashed line) and $\theta\left(\tau^{*}\right) \approx 1-0.86 \exp \left(-4.2 \tau^{*}\right)$ when $\tau^{*} \gtrsim 0.2$ (dotted line). More generally, when $\tau^{*} \ll 1$, for $T_{\mathrm{ph}} \neq 0$,

$$
\theta\left(\tau^{*}\right) \approx \sqrt{\theta^{2}(0)+2\left(1+\theta_{\mathrm{ph}}^{5}-\theta^{5}(0)\right) \tau^{*}} .
$$

Even though $v \gg 1$, we now estimate cooling by electron diffusion to the connecting leads. Starting from a constant temperature $T_{0}$, cooling by diffusion follows Eq. (13) with $a_{k}(\tau)=\left(\frac{k_{B} T_{0}}{e V}\right)^{2} \frac{4}{\pi} \frac{e^{-\pi^{2} k^{2} \tau}}{k}$, and $T_{\mathrm{av}} / T_{0} \sim$ $\exp (-5 \tau)$. Because of this exponential dependence, to be compared with the powerlaw (22), diffusion can contribute to the cooling when $t$ becomes comparable to $\tau_{D}$.

\section{Numerical application}

In experiments where the voltage is applied in repeated pulses, heating is reduced, and the temperature oscillates in time. To illustrate this effect, we reconsider the second example of section IIC, but we now assume that the voltage is applied during short pulses of duration $t_{p}=0.1 \mu \mathrm{s}$, repeated every period $t_{r}=20 \mu \mathrm{s}$
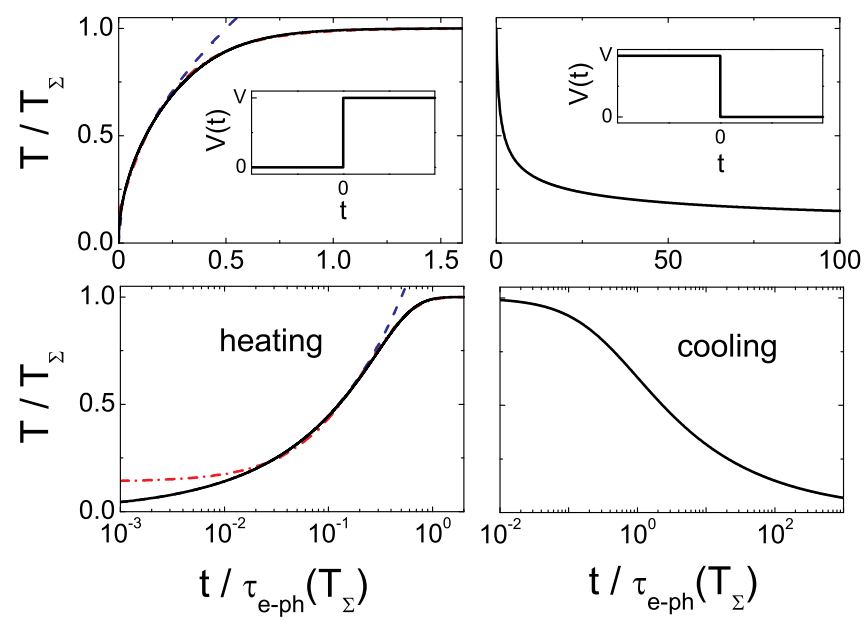

FIG. 6: (Color online) Evolution of the temperature with time in linear (top) and log (bottom) scale in the limit $v \gg 1$, for $T_{p h}=0$. Left panels: heating sequence of the resistor when $V(t) / V=H(t)$ (as shown in inset); right panels: cooling sequence of the resistor when $V(t) / V=1-H(t)$ (as shown in inset). Times are given in units of the electron-phonon time at temperature $T_{\Sigma}: \tau_{e-p h}\left(T_{\Sigma}\right)=\gamma /\left(\Sigma T_{\Sigma}^{3}\right)$. Blue dashed line is $\sqrt{2 \tau^{*}}$, red dashed-dotted line is $1-0.86 \exp \left(-4.2 \tau^{*}\right)$, with $\tau^{*}=t / \tau_{e-p h}\left(T_{\Sigma}\right)$. (which corresponds to actual experimental conditions in Ref. 27, 28 ). We now show that despite the short duty cycle $d=t_{p} / t_{r}=0.005$, heating is not negligible. In our example, $v \sim 30$, therefore the relevant timescale when $V$ is applied is $\tau_{\mathrm{e}-\mathrm{ph}}\left(T_{\Sigma}\right)=\gamma /\left(\Sigma T_{\Sigma}^{3}\right) \sim 10 \mu \mathrm{s}$. Equation (23) with $\theta_{\mathrm{ph}}=15 \mathrm{mK} / T_{\Sigma}=0.1$ gives $T\left(t_{p}\right)=$ $0.17 T_{\Sigma}=25 \mathrm{mK}$, indicating slight heating by the first pulse. The resistance then cools down during a time $t_{r}$ before the next pulse is applied, following Eq.(21), to $T=0.169 T_{\Sigma}$, hardly less than at the end of the first pulse ${ }^{34}$. The temperature rises further during the next pulses, till steady oscillations establish. The full time evolution of $T$ shown in Fig. 7 is obtained by iterating Eq.(21). At each pulse, the temperature rise gets smaller than during the preceeding pulse, because the starting temperature is larger and the heat transfer to phonons becomes more efficient. For the same reason, the cooling between the pulses gets more and more efficient. At $t \gtrsim 250 \mu \mathrm{s}$, a stationary regime is reached, with the reduced temperature oscillating between $\theta_{\text {min }}$ to $\theta_{\max }$ such that $\int_{\theta_{\min }^{2}}^{\theta_{\max }^{2}} \mathrm{~d} w /\left(1+\theta_{\mathrm{ph}}^{5}-w^{5 / 2}\right)=2 \tau_{p}^{*}$ and $\int_{\theta_{\max }^{2}}^{\theta_{\min }^{2}} \mathrm{~d} w /\left(\theta_{\mathrm{ph}}^{5}-w^{5 / 2}\right)=2\left(\tau_{r}^{*}-\tau_{p}^{*}\right)$. One obtains $\theta_{\min }=$ $0.33\left(T_{\min }=49 \mathrm{mK}\right)$ and $\theta_{\max }=0.36\left(T_{\max }=54 \mathrm{mK}\right)$. The amplitude of the oscillations $\Delta \theta=\theta_{\max }-\theta_{\min }$ is therefore very small. However, it increases with $t_{p}$, as shown in the inset of Fig.7, and can become sizeable.

The main features of the time evolution of the temperature can be calculated more simply, from the average Joule power $d \times V^{2} / R$. Using section II, the characteristic temperature is then $T_{\Sigma}^{\text {eff }}=52 \mathrm{mK}$, which fits with the average temperature in the stationary regime of the pulse sequence. According to section IIIB, this temperature is reached in a time $\tau_{\mathrm{e}-\mathrm{ph}}\left(T_{\Sigma}^{\mathrm{eff}}\right) \sim 220 \mu \mathrm{s}$. The rise of temperature with time calculated with Eq. (21), in which all quantities $\left(\theta, \tau^{*}\right)$ are calculated using $T_{\Sigma}^{\text {eff }}$, is shown as a dotted line in Fig.77, and reproduces well the overall behavior. The amplitude $\Delta \theta$ of the temperature oscillations can be evaluated using Eq. (23) under the assumption that the starting temperature is $T_{\Sigma}^{\mathrm{eff}}$, which is a good approximation for oscillations of small amplitude, and considering that the voltage $V$ is always present during the pulse of duration $t_{p}$, i.e. with $\theta(0)=T_{\Sigma}^{\text {eff }} / T_{\Sigma}=d^{1 / 5}$ and $\tau^{*}=t_{p} / \tau_{\mathrm{e}-\mathrm{ph}}\left(T_{\Sigma}\right)$. If $\theta_{\mathrm{ph}} \ll 1$ and $d \ll 1$, one obtains $\Delta \theta \approx \theta(0) \tau^{*}$, an approximation only $20 \%$ larger than the exact result in the worst case of the inset of Fig. $7\left(t_{p}=2 \mu \mathrm{s}\right)$.

\section{SUMMARY}

The solution of the heat equation in a resistor is determined by a characteristic temperature $T_{\Sigma}=$ $\left(V^{2} / \Sigma \Omega R\right)^{1 / 5}$. If $v=e V / k_{B} T_{\Sigma} \lesssim 1$, cooling by phonons is negligible and $T(x)$ is given by Eq. (3), the average temperature by $T_{\mathrm{av}}=\sqrt{3} / 8\left(\mathrm{eV} / k_{B}\right)$. If $v \gtrsim 10$, the temperature is $\left(T_{\Sigma}^{5}+T_{\mathrm{ph}}^{5}\right)^{1 / 5}$ except at distances shorter than 
$\sim 5 L / v$ from the ends. At $v \gtrsim 4$ and $T_{\mathrm{ph}}=0$, the average temperature is $T_{\mathrm{av}} \approx T_{\Sigma}(1-1.16 / v)$. Using these results, we have calculated the decay of the Fano factors $F_{2}$ and $F_{3}$ relative to the second and third cumulants of current fluctuations with the resistor length $L$. We have also addressed time-dependent situations to describe the heating and cooling of resistors. If $v \lesssim 1$, the characteristic timescale is the diffusion time $\tau_{D}$ and heating follows $k_{B} T_{\mathrm{av}}(t) \simeq \frac{\sqrt{3}}{8} \mathrm{eV} \sqrt{1-\exp (-10 \tau)}$, cooling $k_{B} T_{\mathrm{av}}(t) \simeq$ $\frac{\sqrt{3}}{8} e V \exp (-5 \tau)$, with $\tau=t / \tau_{D}$. If $v \gg 1$, the instantaneous relaxation time is $\tau_{\mathrm{e}-\mathrm{ph}}(T)=\gamma /\left(\Sigma T^{3}\right)$. Heating from $T(0)$ to $T_{\Sigma}$ is achieved in a time $\tau_{\mathrm{e}-\mathrm{ph}}\left(T_{\Sigma}\right)$, following $\theta\left(\tau^{*}\right) \approx \sqrt{\theta(0)^{2}+2\left(1+\theta_{\mathrm{ph}}^{5}-\theta(0)^{5}\right) \tau^{*}}$ at short times and $\theta\left(\tau^{*}\right) \approx 1-0.86 \exp \left(-4.2 \tau^{*}\right)$ at long times, with $\theta=T / T_{\Sigma}$ and $\tau^{*}=t / \tau_{\mathrm{e}-\mathrm{ph}}\left(T_{\Sigma}\right)$. Cooling from a temperature $T_{0}$ occurs very slowly, along a powerlaw: at $T_{\mathrm{ph}}=0, T(t) / T_{0}=\left(1+3 t / \tau_{\mathrm{e}-\mathrm{ph}}\left(T_{0}\right)\right)^{-1 / 3}$.

Finally, we recall that the actual temperature can be higher than the predictions made here for at least two reasons. First, the electronic temperature can be larger than $T_{\mathrm{ph}}$ in the connecting wires because of their finite resistivity $\frac{13,22}{2}$ or because of imperfect thermalization to the cryogenic unit. Second, we have neglected the Kapitza resistance $\stackrel{10}{ }$, due to which the phonon temperature inside the resistor can differ from the bath temperature $T_{\mathrm{ph}}$. However, this latest effect is relatively less important in very thin resistors because the ratio of the heat flow from electrons to resistor phonons to the heat

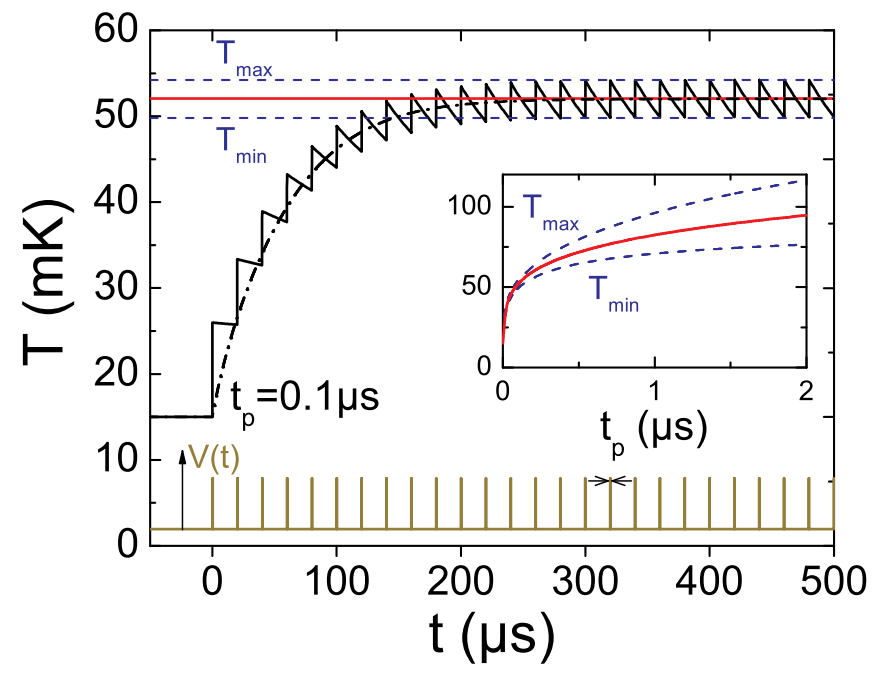

FIG. 7: (Color online) Main panel: Time-dependence of the temperature of a commercial macroscopic surface mount $500 \Omega$ resistor (see text) heated by voltage pulses (bottom curve) of length $t_{p}=0.1 \mu \mathrm{s}$ applied every $20 \mu \mathrm{s}$. Dasheddotted line is the predicted heating with the average Joule power. Inset: (blue dashed lines) minimal and maximal temperature reached in the stationary regime as a function of the pulse length $t_{p}$. In red solid line, temperature evaluated with the average Joule power. flow from resistor phonons to substrate is proportional to the film thickness ${ }^{3}$.

\section{APPENDIX: CALCULATION OF THE THIRD CUMULANTS OF CURRENT IN PRESENCE OF ELECTRON-PHONON SCATTERING}

The calculation of the third cumulant of current in presence of electron-phonon scattering is an extension of the expressions of Pilgram et al $\stackrel{17}{*}$. The third cumulant at zero frequency is expressed as a function of the correlator between temperature and current fluctuations:

$$
S_{3}=\frac{6 k_{B}}{R} \int_{0}^{1} d x\langle\delta T(x) \delta I\rangle .
$$

To calculate the integrand, we start from the stochastic diffusion equation for the fluctuations $\delta f$ of the electron energy distribution function

$$
\begin{aligned}
& \left(\frac{\partial}{\partial t}-\frac{1}{\tau_{D}} \frac{\partial^{2}}{\partial x^{2}}\right) \delta f-\delta I_{e e}-\delta I_{e-p h} \\
& =-e \delta \dot{\phi} \frac{\partial f}{\partial \varepsilon}-\frac{1}{L} \frac{\partial}{\partial x} \delta F^{i m p}-\delta F^{e e}
\end{aligned}
$$

with $\delta I_{e e}$ the linearized electron-electron diffusion integral, $\delta I_{e-p h}$ the linearized electron-phonon diffusion integral, $\delta F^{i m p}$ and $\delta F^{e e}$ random extraneous sources associated with electron-impurity and electron-electron scattering. The correlation function of extraneous sources is

$$
\begin{aligned}
\left\langle\delta F^{i m p}(\varepsilon, x) \delta F^{i m p}\left(\varepsilon^{\prime}, x^{\prime}\right)\right\rangle_{\omega} & =2 \frac{D}{\nu_{F} \Omega} \delta\left(x-x^{\prime}\right) \delta\left(\varepsilon-\varepsilon^{\prime}\right) \\
& \times f(\varepsilon, x)[1-f(\varepsilon, x)] . \quad(26)
\end{aligned}
$$

The energy distribution function is assumed to have a Fermi shape with coordinate dependent temperature $T(x)$ and electrical potential $\phi(x)$ :

$$
f(\varepsilon, x)=\left[1+\exp \left(\frac{\varepsilon-e \phi(x)}{k_{B} T(x)}\right)\right]^{-1} .
$$

To derive the correlator $\left\langle\delta T_{e}(x) \delta I\right\rangle_{\omega}$, Eq. (25) is multiplied by $\varepsilon$ and integrated over energy 22 , assuming that the rate of energy dissipation associated with electronphonon scattering is of the form

$$
\nu_{F} \int d \varepsilon \varepsilon I_{e-p h}=\Sigma\left[T^{5}(x)-T_{p h}^{5}\right] .
$$

The electron-electron collision integral and the associated extraneous source drop out because of energy conservation, and one obtains

$$
\begin{aligned}
& \left(\frac{\partial}{\partial t}-\frac{1}{\tau_{D}} \frac{\partial^{2}}{\partial x^{2}}\right)\left(e^{2} L_{o} T \delta T\right)+5 \nu_{F}^{-1} \Sigma T^{4} \delta T- \\
& -\frac{1}{\tau_{D}} \frac{\partial^{2}}{\partial x^{2}}\left(e^{2} \phi \delta \phi\right)=-\frac{1}{L} \int d \varepsilon \varepsilon \frac{\partial}{\partial x} \delta F^{i m p}
\end{aligned}
$$


Now we multiply Eq. (29) and the equation for the fluctuations of the total current, which in the low-frequency limit reads 18

$$
\delta I=\frac{e \nu_{F} \Omega}{L} \int d \varepsilon \int d x \delta F^{i m p} .
$$

Upon averaging, it gives in the low-frequency limit

$$
\begin{aligned}
& \frac{\partial^{2}}{\partial x^{2}}\left[L_{o} T\langle\delta T(x) \delta I\rangle_{\omega}\right]-L_{o} \alpha T^{4}\langle\delta T(x) \delta I\rangle_{\omega} \\
& =-\frac{\partial^{2}}{\partial x^{2}}\left[\phi\langle\delta \phi(x) \delta I\rangle_{\omega}\right]+\frac{2}{e} \frac{\partial}{\partial x} \int d \varepsilon \varepsilon f(1-f) .
\end{aligned}
$$

with $\alpha=5 \Sigma \Omega R / L_{o}$. The right-hand side of this equation was calculated in Ref.17. The solution of this equation may be written in a symbolic form as

$$
\begin{aligned}
\langle\delta T(x) \delta I\rangle_{\omega} & =\frac{2 k_{B}}{L_{o} T}\left(\frac{\partial^{2}}{\partial x^{2}}-\alpha T^{3}\right)^{-1} \times \\
& \times\left\{\frac{\partial(\phi T)}{\partial x}-\frac{\partial^{2}}{\partial x^{2}}\left[\phi\left(\frac{\partial^{2}}{\partial x^{2}}\right)^{-1} \frac{\partial T}{\partial x}\right]\right\},
\end{aligned}
$$

where the symbol $\left(\partial^{2} / \partial x^{2}-f\right)^{-1}$ is the Green's function $G(x, y)$ such that $\left(\partial^{2} / \partial x^{2}-f\right) G(x, y)=\delta(x-y)$ and $G(0, y)=G(x, 0)=G(1, y)=G(x, 1)=0$. Using $\phi=$ $-V x$, the expression in brackets greatly simplifies:

$$
\left\{\frac{\partial(\phi T)}{\partial x}-\frac{\partial^{2}}{\partial x^{2}}\left[\phi\left(\frac{\partial^{2}}{\partial x^{2}}\right)^{-1} \frac{\partial T}{\partial x}\right]\right\}=V\left(T-2 T_{\mathrm{av}}\right) .
$$

To calculate the third cumulant of the current, one has to solve Eq. (32) and substitute the solution into Eq. (24). The generalized Fano factor $F_{3}=S_{3} / e^{2} I$ is then

$$
\begin{aligned}
F_{3} & =\frac{36}{\pi^{2}} \int_{0}^{1} \mathrm{~d} x \frac{1}{T(x)}\left(\frac{\partial^{2}}{\partial x^{2}}-\alpha T^{3}(x)\right)^{-1}\left\{T-2 T_{\mathrm{av}}\right\} \\
& =\frac{36}{\pi^{2}} \int_{0}^{1} \mathrm{~d} x \mathrm{~d} y \frac{1}{\theta(x)} G_{1}(\theta, x, y)\left\{\theta(y)-2 \theta_{\mathrm{av}}\right\}
\end{aligned}
$$

with $G_{1}(\theta, x, y)$ the Green's function such that

$$
\left(\frac{\partial^{2}}{\partial x^{2}}-\frac{15}{\pi^{2}} v^{2} \theta^{3}(x)\right) G_{1}(\theta, x, y)=\delta(x-y),
$$

which can be calculated from ${ }^{21}$

$$
G_{0}(x, y)=\left(\frac{\partial^{2}}{\partial x^{2}}\right)^{-1}=\min (x, y)(\max (x, y)-1)
$$

using

$$
G_{1}=\left(1-\frac{15}{\pi^{2}} v^{2} G_{0} \theta^{3}(x)\right)^{-1} G_{0}
$$

In practice, we performed this calculation by discretization of the coordinates and matrix inversion: the resistor is cut into $N$ pieces of length $\varepsilon=1 / N$, and the function $G_{0}(x, y)$ is represented with a matrix $G^{0}$ such that

$$
G_{i j}^{0}=-\frac{\varepsilon}{N} \min (i, j)(\max (i, j)-1)
$$

$(0 \leq i, j \leq N)$. The term $\frac{15}{\pi^{2}} v^{2} G_{0} \theta^{3}(x)$ is represented by the matrix $F$ build on the calculated temperature profile $\theta(x)$ using

$$
F_{i j}=G_{i j}^{0} \times \frac{15}{\pi^{2}} v^{2} \theta^{3}(j \varepsilon) .
$$

We then invert the matrix $A$ with $A_{i j}=\frac{1}{\varepsilon} \delta_{i j}-F_{i j}$ and compute $G^{1}=A^{-1} \cdot G^{0}$. Finally,

$$
F_{3}=\frac{36}{\pi^{2}} \varepsilon \sum_{i, j} \frac{G_{i j}^{1}\left\{-\theta(j \varepsilon)+2 \theta_{\mathrm{av}}\right\}}{\theta(i)} .
$$

\section{Acknowledgments}

F. Pierre, Norman O. Birge and A. Anthore were involved in the early stages of this work. Discussions with B. Reulet, H. Grabert, Yu. Gefen and within the Quantronics group are gratefully acknowledged. We particularly appreciated guidance from Hermann Grabert in the implementation of the calculation of the third cumulant of noise. This work was partly funded by the Agence Nationale de la Recherche under contract ANR05-NANO-039.
1 M.R. Arai, Appl. Phys. Lett. 42, 906 (1983).

2 M.L. Roukes, M.R. Freeman, R.S. Germain, R.C. Richardson and M.B. Ketchen, Phys. Rev. Lett. 55, 422 (1985).

3 F.C. Wellstood, C. Urbina and J. Clarke, Phys. Rev. B 49, 5942 (1994).

4 F. Pierre, A.B. Gougam, A. Anthore, H. Pothier, D. Esteve, and Norman O. Birge, Phys. Rev. B 68, 085413 (2003).
5 A. Sergeev and V. Mitin, Phys. Rev. B 61, 6041 (2000).

${ }^{6}$ Andrew H. Steinbach, John M. Martinis and Michel H. Devoret, Phys. Rev. Lett. 76, 3806 (1996).

7 Francesco Giazotto, Tero T. Heikkilä, Arttu Luukanen, Alexander M. Savin, and Jukka P. Pekola, Rev. Mod. Phys. 78, 217 (2006).

${ }^{8}$ H. Pothier, S. Guéron, N.O. Birge, D. Esteve, and M.H. Devoret, Phys. Rev. Lett. 79, 3490 (1997). 
9 B. Huard, A. Anthore, Norman O. Birge, H. Pothier, and D. Esteve, Phys. Rev. Lett. 95, 036802 (2005).

10 E. T. Swartz and R. O. Pohl, Rev. Mod. Phys. 61, 605 (1989).

11 D. R. Schmidt, R. J. Schoelkopf, and A. N. Cleland, Phys. Rev. Lett. 93, 045901 (2004).

12 Matthias Meschke, Wiebke Guichard, and Jukka P. Pekola, Nature 444, 187 (2006).

13 M. Henny, S. Oberholzer, C. Strunk, and C. Schönenberger, Phys. Rev. B 59, 2871 (1999).

14 More rigourously, from Eq. (3), one obtains $13 T_{\mathrm{av}} / T_{\mathrm{ph}}=$ $\frac{1}{2}\left(1+\left(u+\frac{1}{u}\right) \arctan u\right)$ with $u=\sqrt{3} e V / 2 \pi k_{B} T_{\mathrm{ph}}$. This expression differs from $\left(1+(\pi u / 4)^{2}\right)^{1 / 2}$ by at most $2.5 \%$.

15 At $T_{\mathrm{ph}}=0$ and $x v \ll 1, \frac{T(x)}{T_{\Sigma}} \simeq \sqrt{a x v}$, with $a=\frac{2}{\pi} \sqrt{\frac{15}{7}} \simeq$ 0.93 .

${ }^{16}$ We have corrected the erroneous prefactor in Ref. 6 .

17 S. Pilgram, K.E. Nagaev and M. Büttiker, Phys. Rev. B 70, 045304 (2004).

18 K.E. Nagaev, Phys. Lett. A 169, 103 (1992).

19 C. W. J. Beenakker and M. Büttiker, Phys. Rev. B 46, 1889 (1992).

${ }^{20}$ Hyunwoo Lee, L.S. Levitov, and A.Yu. Yakovets, Phys. Rev. B 51, 4079 (1995).

21 D.B. Gutman and Yuval Gefen, Phys. Rev. B 68, 035302 (2003).

${ }^{22}$ K.E. Nagaev, Phys. Rev. B 52, 4740 (1995).

${ }^{23}$ Y. Naveh, D.V. Averin, and K.K. Likharev, Phys. Rev. B 58, 15371 (1998).
${ }^{24}$ K.E. Nagaev, Phys. Rev. B 66, 075334 (2002).

25 P. Joyez, D. Esteve, and M. H. Devoret, Phys. Rev. Lett. 80, 1956 (1998).

26 B. Huard, Ann. Phys. Fr. 31, N 4-5 (2006).

27 D. Vion, A. Aassime, A. Cottet, P. Joyez, H. Pothier, C. Urbina, D. Esteve, and M.H. Devoret, Science 296, 286 (2002).

28 A. Cottet, PhD Thesis (2002), Université Paris 6 (in English, downloadable at http://tel.archives-ouvertes.fr/tel-00003511/).

29 D. R. Schmidt, C. S. Yung, and A. N. Cleland, Phys. Rev. B 69, 140301(R) (2004).

30 Using a microscopic description of electron-phonon scattering, $\gamma / \Sigma$ can be related to Fermi liquid parameters and to the sound velocity ${ }^{3}$. However, in experiments, a large discrepancy was found with the corresponding theoretical values $3,31,32$.

31 F. Pierre, Ann. Phys. Fr. 26, N 4 (2001).

32 S.-X. Qu, A. N. Cleland, and M. R. Geller, Phys. Rev. B 72, 224301 (2005).

33 We don't retain a factor 5 introduced in the definition of $\tau_{\mathrm{e}-\mathrm{ph}}$ in Ref.11. The length $L_{\mathrm{e}-\mathrm{ph}}$ defined in section IIA is related to $\tau_{\mathrm{e}-\mathrm{ph}}(T)$ by $L_{\mathrm{e}-\mathrm{ph}}=$ $8^{5 / 2} 3^{-3 / 4} \pi^{-1}\left(D \tau_{\mathrm{e}-\mathrm{ph}}\left(\mathrm{eV} / k_{B}\right)\right)^{1 / 2}$.

34 Cooling by diffusion is here ineffective because of the too long diffusion time $\tau_{D} \sim 2 \mathrm{~ms}$.

35 V. I. Kozub and A. M. Rudin, Phys. Rev. B 52, 7853 (1995). 